\title{
Overexpression of long noncoding RNA NORAD in colorectal cancer associates with tumor progression
}

This article was published in the following Dove Press journal:

OncoTargets and Therapy

\author{
Lili Wang' \\ Lutao $\mathrm{Du}^{2}$ \\ Weili Duan ${ }^{2}$ \\ Suzhen Yan $^{2}$ \\ Yujiao Xie ${ }^{2}$ \\ Chuanxin Wang ${ }^{2}$ \\ 'Department of Clinical Laboratory, \\ Qilu Hospital of Shandong University, \\ Jinan, Shandong 2500 I2, People's \\ Republic of China; ${ }^{2}$ Department \\ of Clinical Laboratory, The Second \\ Hospital of Shandong University, Jinan, \\ Shandong 250033, People's Republic \\ of China
}

Purpose: The aim of this study was to elucidate the role and clinical significance of long noncoding RNA-activated by DNA damage (NORAD) in colorectal cancer (CRC).

Methods: Sixty pairs of tumorous and adjacent nontumorous tissues derived from CRC patients were subjected to quantitative real-time polymerase chain reaction to determine the expression level of NORAD. The serum levels of NORAD expression were also measured in an independent cohort of CRC patients as well as patients with benign diseases and healthy controls. Comparative analyses were performed to investigate the relationships between NORAD levels in tissues and clinicopathological features of CRC. Receiver operating characteristic (ROC) curve analysis was used to assess the diagnostic value of NORAD in patients with CRC. Furthermore, the potential functions of NORAD in the development of CRC were explored in vitro, using the HCT116 and SW1116 CRC cell lines.

Result: NORAD expression was significantly upregulated in the tumorous tissues of CRC patients $(P<0.001)$ compared to the adjacent nontumorous tissues. Higher NORAD expression was associated with advanced CRC. Moreover, serum levels supported that NORAD could distinguish CRC patients from healthy controls and patients with benign diseases, indicating a potential diagnostic role in CRC. The ROC curve analysis showed a diagnostic efficacy with area under the curve of 0.800 (95\% CI: 0.737-0.853). Mechanistic investigations indicated that NORAD silencing reduced CRC cell proliferation, migration, and invasion.

Conclusion: NORAD may serve as a novel predictor in $\mathrm{CRC}$ and may be a potential target for future therapy.

Keywords: biomarker, long noncoding RNA, noncoding RNA-activated by DNA damage, diagnosis

\section{Introduction}

Colorectal cancer (CRC) is the third most common cancer as well as the fourth most common cause of death from cancer worldwide, with over 1.2 million newly diagnosed cases annually, and the incidence is estimated to increase to 2.4 million by $2035 .^{1,2}$ Despite significant improvements in the diagnostic technologies and therapeutic strategies, CRC retains an overall poor prognosis and recurrent disease rates remain high, with 5 -year survival rates being only $10 \%-15 \% .^{3,4}$ Therefore, studies to increase our understanding of the molecular mechanisms that result in CRC and explore potential genetic biomarkers of this disease are urgently needed to support efforts that will improve clinical outcomes.
Correspondence: Chuanxin Wang Department of Clinical Laboratory, The Second Hospital of Shandong University, 247 Beiyuan Street, Jinan 250033,

People's Republic of China Fax +86 53I 88962544

Email cxwang@sdu.edu.cn 
Long noncoding (lnc) RNAs are commonly longer than 200 nucleotides in length, having limited or no potential ability for protein coding. ${ }^{5,6}$ A multitude of lncRNAs have been implicated in cancer biology, carcinogenesis, and metastasis. ${ }^{7-10}$ Moreover, investigations into their functional mediation of oncogenes, tumor suppressor genes, or both have provided new insights into the underlying mechanisms of various cancers, including CRC. For example, the lncRNA MALAT1 was shown to play an important role in tumorigenesis and the metastatic cascade of $\mathrm{CRC}^{11}$ and the lncRNA HOTAIR was demonstrated to be upregulated in primary tumors and in blood of CRC patients, being associated with unfavorable prognosis. ${ }^{12}$ In addition, Su et a ${ }^{13}$ characterized the lncRNA BLACAT1 as a cell cycle regulator, affecting cell proliferation by epigenetic silencing of $\mathrm{p} 15$.

Recently, a novel conserved lncRNA named "noncoding RNA-activated by DNA damage" (NORAD) has been characterized. NORAD is also known as LINC00657 and is located on chromosome 20 with a length of $5.3 \mathrm{~kb}$ and induced in response to DNA damage. NORAD is abundant with approximately $500-1,000$ copies expression levels per cell in human cell lines. It has been shown that NORAD loss-of-function mutation can regulate genome stability by modulating PUMILIO proteins in the cytoplasm, which have a potential repression role in the process of DNA repair and replication. ${ }^{14,15}$ Furthermore, Tichon et $\mathrm{a}^{16}$ demonstrated that the RNA-binding protein SAM68 (KHDRBS1) is required for the PUMILIO antagonizing activity of NORAD. Furthermore, NORAD has been characterized as a potential oncogene in various cancers. ${ }^{17-19} \mathrm{Li}$ et a ${ }^{17}$ recently demonstrated that NORAD is overexpressed in pancreatic cancer tissues, wherein it serves as a novel competing endogenous RNA to promote epithelial-mesenchymal transition and metastasis by regulating RhoA in a microRNA 125a-3p-dependent manner. However, to the best of our knowledge, the potential role of NORAD and the clinical significance of its circulating expression level in CRC remain unknown.

In the present study, we investigated the level of NORAD in tumor tissues compared to that in adjacent noncancerous tissues of CRC patients and investigated the potential relationships between NORAD expression and clinicopathological features. The serum levels of NORAD were also examined in the CRC patients and compared with those in healthy subjects and patients with benign diseases. Finally, the functional roles of NORAD in the molecular tumorigenic mechanisms of CRC were explored in vitro to provide foundational knowledge for their potential for future therapeutic application.

\section{Materials and methods Patient and tissue specimens}

A total of 60 resected tumor tissues and adjacent noncancerous tissues (at least $5 \mathrm{~cm}$ away from the tumor margin) were obtained from CRC patients, with written informed consent from each patient or legal representative, in the Department of General Surgery, Qilu Hospital of Shandong University. Serum samples were also obtained from an independent cohort composed of 142 CRC patients, 136 healthy controls, and 71 patients with benign diseases including inflammatory bowel disease, hyperplastic disease, and adenoma. No patient received any preoperative treatment. The study was carried out with approval by the ethics committee of Qilu Hospital of Shandong University.

In all cases of CRC, diagnosis was histopathologically confirmed, and demographic and clinical data were collected, including age, sex, tumor location, tumor size, differentiation, lymph node metastasis, and distant metastasis. All resected tissue samples were snap-frozen in liquid nitrogen immediately upon obtainment and stored at $-80^{\circ} \mathrm{C}$ until subject to RNA extraction. The postoperative pathologic staging was performed according to the 7th edition of the Union for International Cancer Control tumor-node-metastasis (commonly known as TNM) staging system. Venous blood samples were collected and stored at $-80^{\circ} \mathrm{C}$ until analysis.

\section{Cell culture}

The human CRC cell lines HCT116 and SW1116 (American Type Culture Collection, Manassas, VA, USA) were cultured in Roswell Park Memorial Institute 1640 (Thermo Fisher Scientific, Waltham, MA, USA) containing $10 \%$ fetal bovine serum (FBS) (Sigma-Aldrich Co., St Louis, MO, USA) and were maintained at $37^{\circ} \mathrm{C}$ in a $5 \% \mathrm{CO}_{2}$ atmosphere.

\section{Total RNA extraction and quantitative real-time (q)PCR}

Total RNA was isolated from the primary human tissues or cells using Trizol reagent (Thermo Fisher Scientific) according to the manufacturer's instructions. Quantity and quality of the isolated RNA were determined using NanoDrop 2000 (Thermo Fisher Scientific) prior to application for cDNA synthesis using the Prime Script ${ }^{\mathrm{TM}}$ RT Reagent Kit (Takara, Dalian, People's Republic of China). The samples, with reaction volume of $20 \mu \mathrm{L}$ consisting of $1 \mu \mathrm{g}$ of template RNA, $4 \mu \mathrm{L}$ of $5 \times$ Prime Script buffer mix, $1 \mu \mathrm{L}$ of oligo $\mathrm{dT}$ primer, and $1 \mu \mathrm{L}$ of Prime Script RT enzyme mix, were first incubated at $37^{\circ} \mathrm{C}$ for 30 minutes, followed by $85^{\circ} \mathrm{C}$ for 5 seconds. Then, qPCR was performed with SYBR Premix 
ExTaq $^{\text {TM }}$ (Takara) on the CFX96TM Real-Time System (Bio-Rad Laboratories Inc., Hercules, CA, USA) with a thermocycling profile of $95^{\circ} \mathrm{C}$ for 30 seconds, followed by 40 cycles of $95^{\circ} \mathrm{C}$ for 5 seconds and $60^{\circ} \mathrm{C}$ for 34 seconds. Specificity of the qPCR products was evaluated by melting curve analysis and confirmed by sequencing. The relative expression of NORAD was calculated using the comparative cycle threshold $\left(C_{t}\right)\left(2^{-\Delta \Delta C t}\right)$ method. The primer sequences of lncRNA NORAD used in this study are listed in Table S1.

\section{Knockdown of NORAD in CRC cells}

CRC cells were transfected with three siRNAs specifically targeting lncRNA NORAD (si-NORAD [no 1, no 2, and no 3], 100 nM each; Ribobio, Guangzhou, People's Republic of China) or negative control siRNA (si-NC; Ribobio), which was performed using Lipofectamine 2000 (Thermo Fisher Scientific) according to the manufacturer's instructions. Knockdown effect of si-NORAD was examined by RT-qPCR using RNA extracted 48 hours after transfection. The siRNA sequences of IncRNA NORAD and negative control used in this study are listed in Table S1.

\section{Cell viability analysis}

Cell viability was assessed by the Cell Counting Kit- 8 (CCK-8; Beyotime, Hangzhou, People's Republic of China). Briefly, the transfected HCT116 or SW1116 cells were seeded in 96 -well plates at a density of $5 \times 10^{3}$ cells per well, with three replicate wells per group, and cultured for 24,48 , and 72 hours. CCK-8 reagent $(10 \mu \mathrm{L})$ was added to each well and allowed to incubate for an additional 2 hours at $37^{\circ} \mathrm{C}$. The optical density was measured at $450 \mathrm{~nm}$ using a microplate photometer (Multiskan FC; Thermo Fisher Scientific). Experiments were performed independently in triplicate.

\section{Colony formation assays}

CRC cells transfected with si-NC/si-NORAD were collected and seeded in a 6-well plate at a density of 300 cells per well and cultured for 10 days in Roswell Park Memorial Institute 1640 containing $10 \%$ FBS. The cells were then fixed by incubating in 4\% paraformaldehyde for 15 minutes and stained with $0.5 \%$ crystal violet.

\section{Transwell assay}

Transwell chambers with $8 \mu \mathrm{m}$ pores (Corning Costar, Corning, NY, USA) were used to assess cell migration and invasion of the transfected cells. Briefly, at 24 hours posttransfection, the cells were harvested and resuspended into $100 \mu \mathrm{L}$ medium containing 1\% FBS and then seeded into an upper Transwell chamber. Medium containing 10\% FBS was then added to the lower chamber. After incubation for 48 hours at $37^{\circ} \mathrm{C}$, the cells were fixed on the filter surface with methanol, stained with $0.1 \%$ crystal violet, and photographed under magnification (Olympus Corporation, Tokyo, Japan). For cell invasion assay, the Transwell membranes were precoated with matrix gel and the assay proceeded as described above. All assays were performed in triplicate, and cells from at least five microscopic fields were counted by eye.

\section{Statistical analysis}

Data were summarized as mean $\pm \mathrm{SD}$. The levels of NORAD expression were compared between groups using the MannWhitney $U$ test or Kruskal-Wallis test, as appropriate. Receiver operating characteristic (ROC) curve analysis was applied to illustrate the diagnostic value using MedCalc 9.3.9.0 (MedCalc Software, Mariakerke, Belgium). A twosided $P$-value $<0.05$ was considered to indicate statistical significance. Data were analyzed using GraphPad Prism (version 5.0; GraphPad Software, La Jolla, CA, USA).

\section{Results}

NORAD is upregulated in human CRC tumors

qPCR measurement of NORAD expression levels in the 60 matched CRC tissue samples showed significantly higher expression in the tumor tissues than in the adjacent nontumor tissues ( $P=0.024$; Figure $1 \mathrm{~A})$. Subsequent statistical analysis of the levels of NORAD expression indicated associations with various clinicopathological features (Table 1). In particular, the NORAD expression level was significantly higher in patients with advanced $\mathrm{CRC}(P<0.05$; Figure 1B) and those with lymph node metastasis ( $P=0.037$; Figure $1 \mathrm{C})$. In addition, the level of NORAD expression showed significant association with local invasion ( $P=0.014$; Figure 1D). However, the increased level of NORAD expression in CRC tissues was not statistically correlated with sex, age, tumor location, tumor size, or tumor differentiation. The observed upregulation of NORAD in tumor tissues supports its potential involvement in the progression of CRC development.

\section{NORAD is upregulated in the serum of CRC patients}

As shown in Figure 2A, CRC patients showed significantly higher serum level of NORAD (1.495 \pm 1.3024$)$ compared to that in healthy controls $(0.492 \pm 0.681)$ and patients with benign diseases (1.021 \pm 0.975$)$. More importantly, our results demonstrated the potential significance of NORAD 
A
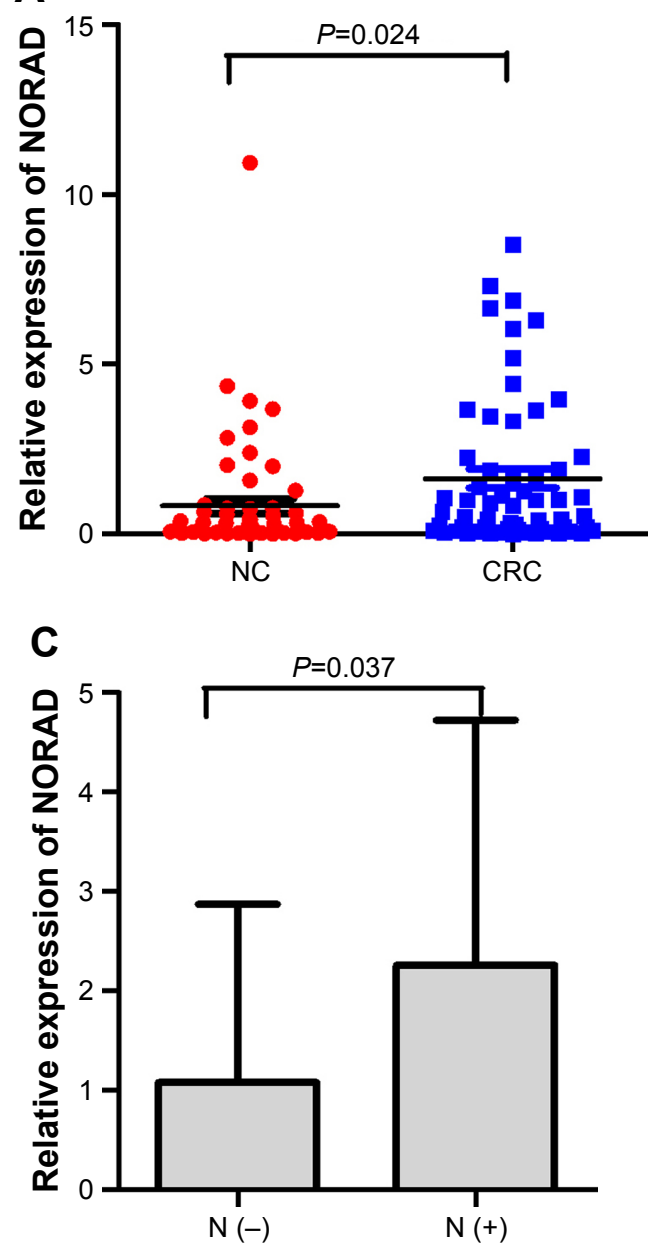

B

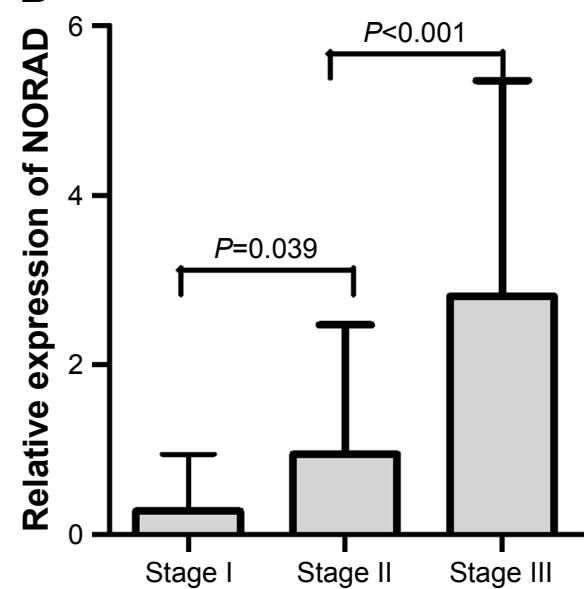

D

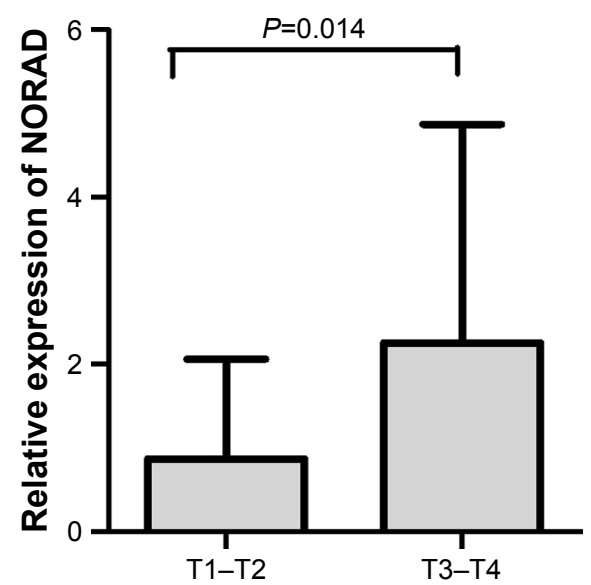

Figure I High NORAD expression is associated with the progression of tumor development in CRC patients.

Notes: (A) NORAD expression was determined by qPCR, normalized against GAPDH, in 60 paired CRC tissues and adjacent noncancerous tissues; (B) the level of NORAD in TNM stages of CRC; (C) the overexpression of NORAD in CRC was associated with lymph node metastasis; (D) increased levels of NORAD were associated with deeper invasion in CRC. CRC diagnosis was confirmed by histopathological method TNM data, metastases and stage were classified according to the TNM classification. Abbreviations: CRC, colorectal cancer; NORAD, noncoding RNA-activated by DNA damage; qPCR, quantitative polymerase chain reaction.

for distinguishing CRC from benign diseases $(P<0.001)$. Moreover, the diagnostic efficacy of NORAD was good, as suggested by area under ROC curve of 0.800 (95\% CI: $7.737-0.853$; Figure $2 B$ ), sensitivity of $81.7 \%$, and specificity of $70.7 \%$.

\section{Downregulation of NORAD suppressed CRC cell viability}

In order to explore the functional role of NORAD in the development and progression of $\mathrm{CRC}$, we first downregulated NORAD by siRNA transfection of HCT116 and SW1116 cells. The knockdown effect is shown in Figure 3A, and we finally chose si-NORAD (no 1) for further experiments. CCK8 assay showed that cells transfected with si-NORAD exhibited reduced cell counts compared with the si-NC control cells at 72 hours in HCT116 and SW1116 cell lines $(P=0.048, P=0.016$, respectively) (Figure 3B and $C)$. However, no significant differences were found at 48 hours $(P>0.05)$. The colony formation experiments indicated a higher suppressive effect in CRC cells transfected with siNORAD compared with those with si-NC control (Figure 3D and $\mathrm{E})$.

\section{Downregulation of NORAD reduced cell migration and invasion}

To determine the effect of NORAD on tumor mobility and invasiveness, we performed Transwell assays with or without Matrigel to explore siRNA-mediated regulation of NORAD in CRC cells. The results showed that decreased expression of NORAD clearly inhibited the migratory capacity of HCT116 cells and SW1116 cells $(P<0.001$; Figure 4A and $P<0.001$; Figure 4B). Similarly, the invasion abilities were 
Table I Relationship between NORAD expression and demographic and clinical characteristics of patients with CRC

\begin{tabular}{|c|c|c|c|}
\hline \multirow[t]{2}{*}{ Characteristics } & \multirow{2}{*}{$\begin{array}{l}\text { Cases } \\
n=60\end{array}$} & \multirow{2}{*}{$\begin{array}{l}\text { Expression level } \\
2^{-\Delta \Delta C t}\end{array}$} & \multirow[t]{2}{*}{$P$-value } \\
\hline & & & \\
\hline Gender & & & 0.128 \\
\hline Male & 34 & $2.01(2.52)$ & \\
\hline Female & 26 & I.I3 (I.57) & \\
\hline Age (years) & & & 0.226 \\
\hline$\leq 60$ & 29 & $1.28(1.66)$ & \\
\hline$>60$ & 31 & $1.97(2.58)$ & \\
\hline Tumor location & & & 0.432 \\
\hline Colon & 20 & I.3I (2.29) & \\
\hline Rectum & 40 & $1.78(2.15)$ & \\
\hline Differentiation & & & 0.517 \\
\hline Well & 8 & $0.866(\mathrm{I} .48)$ & \\
\hline Moderate & 39 & $1.67(2.12)$ & \\
\hline Poor & 13 & $1.99(2.769)$ & \\
\hline Size $(\mathrm{cm})$ & & & 0.604 \\
\hline$\leq 5$ & 36 & $1.75(2.20)$ & \\
\hline$>5$ & 24 & $1.45(2.20)$ & \\
\hline Local invasion & & & $0.014 *$ \\
\hline $\mathrm{TI}-\mathrm{T} 2$ & 27 & $0.87(1.19)$ & \\
\hline T3-T4 & 33 & $2.26(2.61)$ & \\
\hline Lymph node positive metastasis & & & $0.037^{*}$ \\
\hline No & 32 & $1.08(1.79)$ & \\
\hline Yes & 28 & $2.26(2.46)$ & \\
\hline TNM stages & & & $<0.00 I^{*}$ \\
\hline $\mathrm{I}$ & 11 & $0.28(0.67)$ & \\
\hline II & 23 & $0.95(1.52)$ & \\
\hline III & 26 & $2.81(2.53)$ & \\
\hline
\end{tabular}

Note: $* P<0.05$.

Abbreviations: CRC, colorectal cancer; NORAD, noncoding RNA-activated by DNA damage.

A

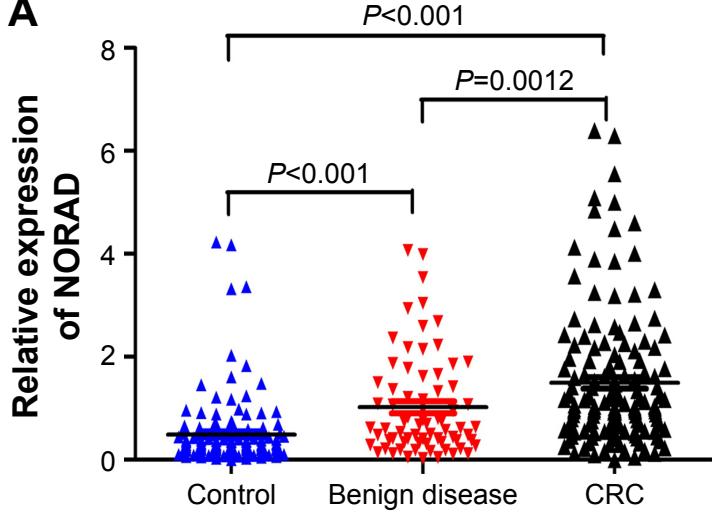

B

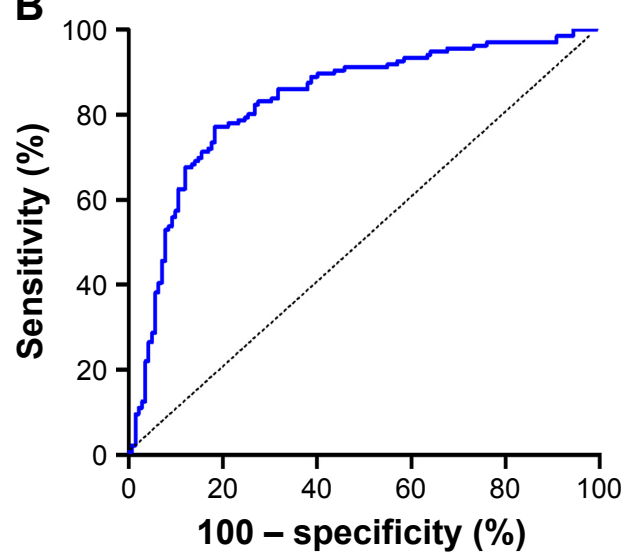

Figure 2 Expression levels of NORAD in sera of healthy controls, patients with CRC, and patients with benign diseases.

Notes: (A) The expression level of IncRNA NORAD was significantly higher in the CRC group than in the healthy control group and benign disease group. (B) ROC curve analysis for the detection of CRC using IncRNA NORAD.

Abbreviations: CRC, colorectal cancer; Inc, long noncoding; NORAD, noncoding RNA-activated by DNA damage; ROC, receiver operating characteristic. 

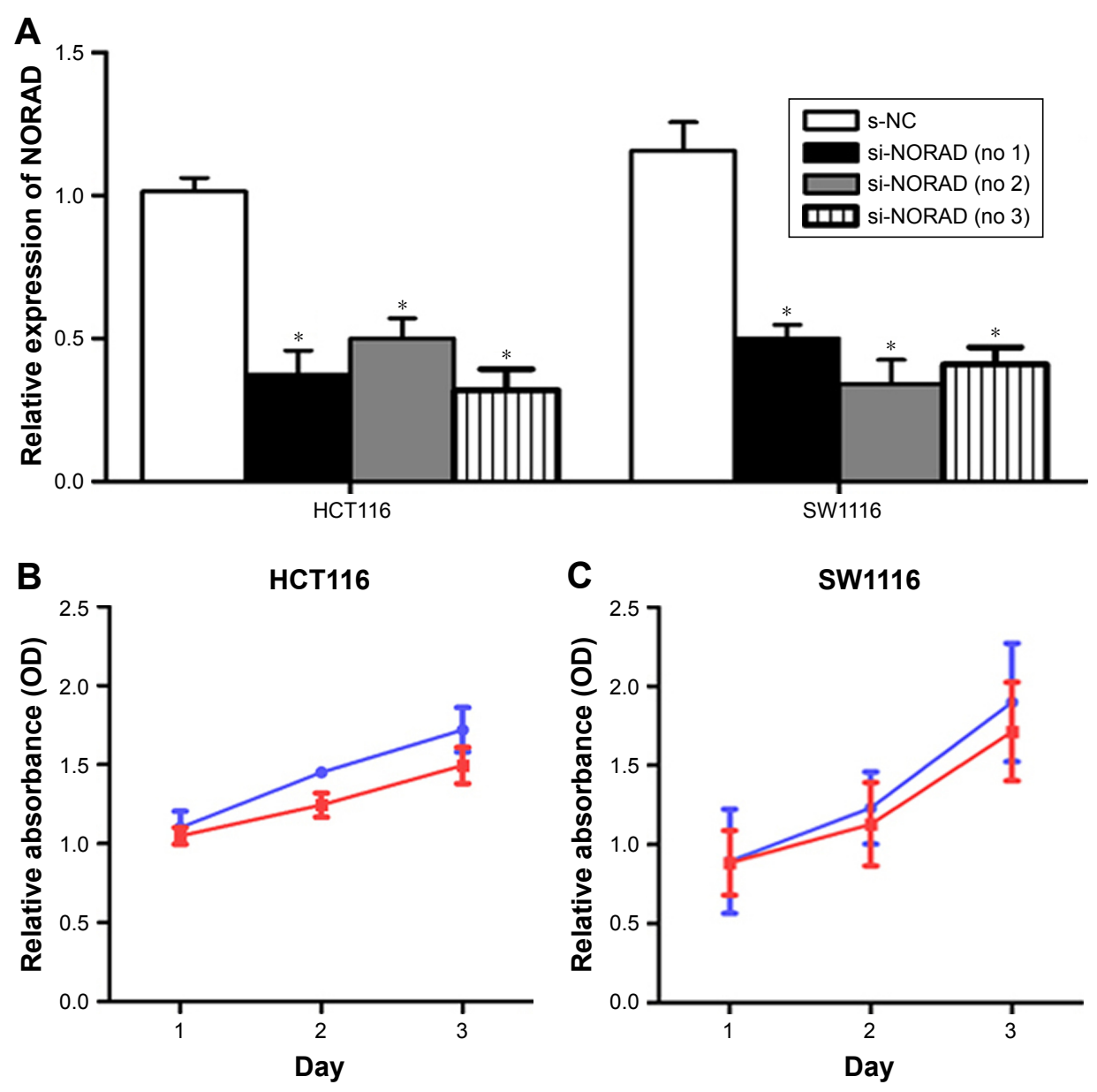

D
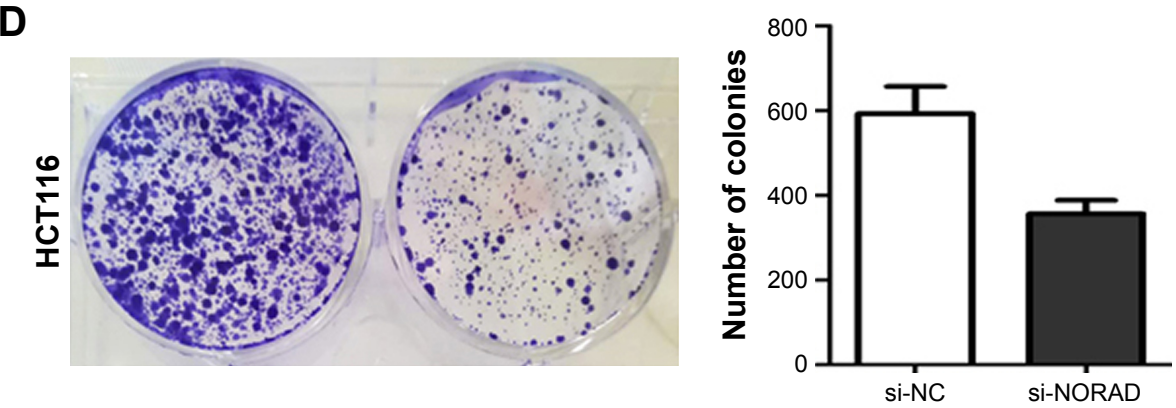

E
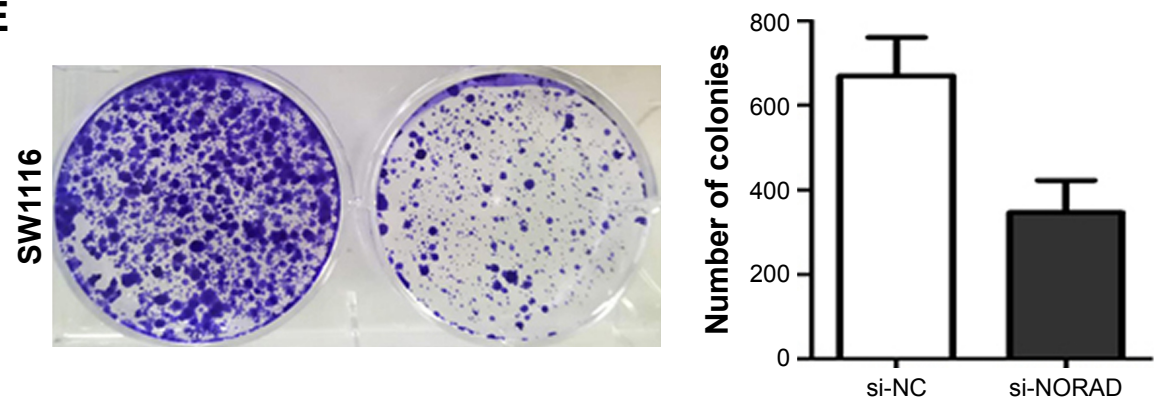

Figure 3 Effect of NORAD knockdown on CRC cell viability in vitro.

Notes: (A) Validation of si-NORAD knockdown effect in HCTII6 and SWIII6. (B and C) Growth curves of si-NC/NORAD-transfected HCTII6 and SWIII6 CRC cells, respectively, were determined by findings from the CCK-8 assay; (D and $\mathbf{E})$ representative photographs and statistical analyses of the colony formation assay of si-NC/ NORAD-transfected CRC cells. $* P<0.05$.

Abbreviations: CCK, Cell Counting Kit; CRC, colorectal cancer; NC, negative control; NORAD, noncoding RNA-activated by DNA damage. 
A
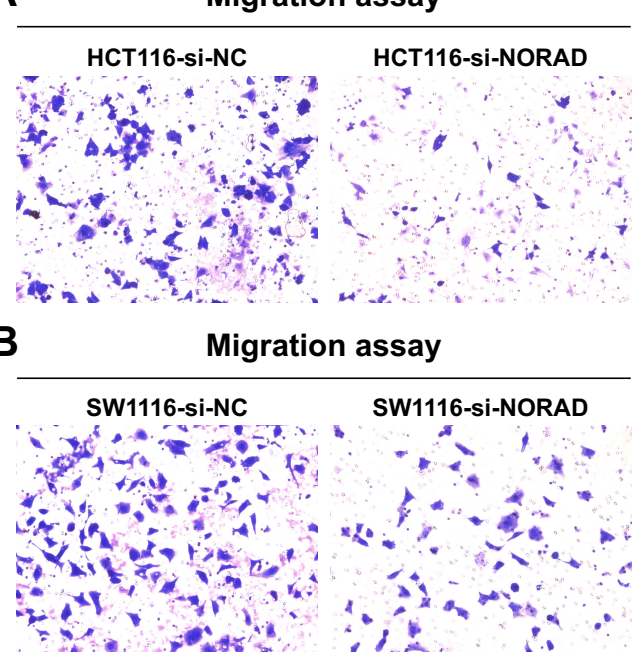

C
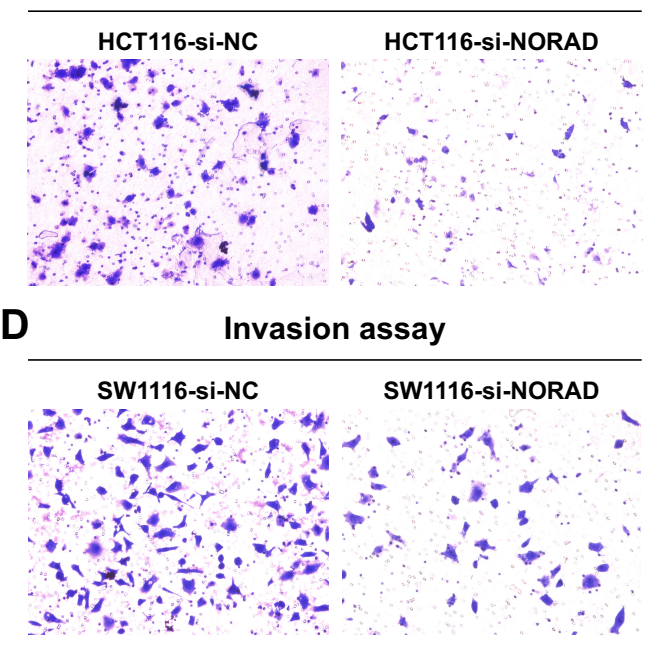
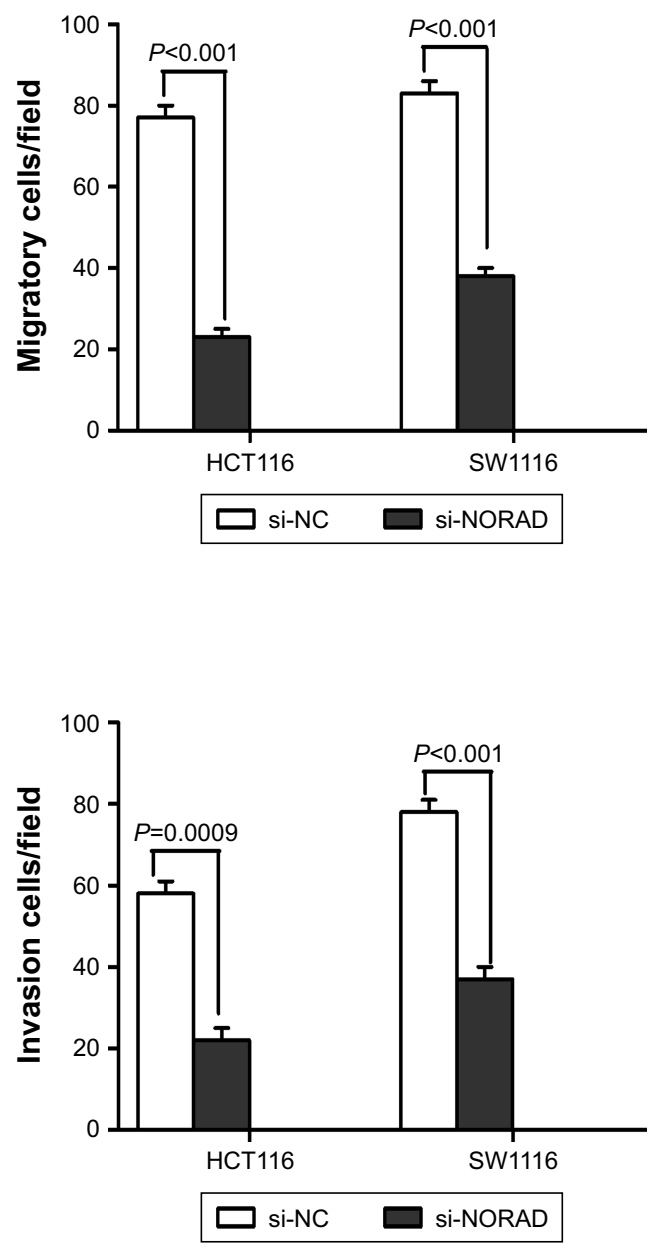

Figure 4 Effect of NORAD knockdown on CRC cell function determined by Transwell assay.

Notes: (A and B) Downregulation of NORAD significantly inhibited the migration of HCTII6 and SWIII6 CRC cells, respectively; (C and D) invasion abilities were suppressed in cells transfected with NORAD siRNA. The representative pictures and statistical analyses involved five randomly selected fields (all $P<0.00 \mathrm{I}$ ).

Abbreviations: CRC, colorectal cancer; NC, negative control; NORAD, noncoding RNA-activated by DNA damage.

significantly suppressed in cells transfected with NORAD siRNA, as compared to the si-NC control transfected cells ( $P=0.0009$; Figure $4 \mathrm{C}$ and $P<0.001$; Figure 4D). The observed significant impairment of migratory and invasive abilities of NORAD-deficient CRC cells suggests that this lncRNA might function as a tumor suppressor in human CRC progression.

\section{Discussion}

The lncRNAs encoded by mammalian genomes are a fascinating class of genes. The approximately $410,000^{3,4}$ mammalian IncRNAs reported to date reflect the increased attention of research aimed at deciphering their underlying roles in various physiological and pathogenic processes, with cancer being a particular focus. While lncRNAs have been demonstrated as regulators of genetic alternation, transcriptional activation, and other processes, ${ }^{6-9}$ numerous studies have shown their important roles in CRC tumorigenesis. The CRC-related lncRNAs characterized to date have been shown to exert their effects by disrupting various biological processes, ${ }^{17-20}$ such as chromatin remodeling, RNA decay, and transcription. These findings suggest the potential of IncRNAs to be developed as effective biomarkers for diagnostic, prognostic, or therapeutic purposes in clinic. In this study, we focused on NORAD (a novel highly abundant and conserved mammalian lncRNA) to determine its role in CRC and its potential value for future clinical applications.

We first analyzed the expression levels of NORAD in 60 human CRC tissue samples. We found that NORAD levels were significantly upregulated in the CRC tissues, as 
compared with adjacent nontumorous tissues. The increased levels of NORAD were associated with deeper invasion, positive lymph node metastasis, and higher TNM stage, suggesting that NORAD might participate in CRC progression. The abovementioned results were also consistent with the findings from Zhang et al. ${ }^{21}$ Moreover, to explore the potential clinical value of NORAD, particularly as a diagnostic biomarker, we compared the expression levels of NORAD in CRC patients, healthy controls, and patients with benign diseases. The results indicated that NORAD expression was dramatically increased in the serum of CRC patients, supporting its potential value in distinguishing benign diseases from CRC. Furthermore, the ROC curve analysis showed good diagnostic efficacy. Overall, these results suggested that NORAD might be developed as a biomarker for CRC diagnosis and minimally invasive tracking of progression.

Research efforts to elucidate the precise mechanisms underlying the initiation and progression of CRC are ongoing. Tumor progression in CRC is a multistep process involving the dysregulation of genetic alterations. ${ }^{22-24}$ Notably, NORAD can negatively regulate the activity of PUMILIO, which might be vital for the initial progression of cancer. In addition, the functions of migration and invasion are necessary for tumor cells spreading to the lymph nodes or blood vessels. To further elucidate the role of NORAD in the development of CRC, we knocked down the expression of NORAD by siRNA in the present study. This downregulation of NORAD significantly suppressed the CRC cell proliferation, migration, and invasion. These results collectively suggest the promise of NORAD as a novel therapeutic target to repress tumor progression.

\section{Conclusion}

Our findings of upregulated expression of NORAD in both tissue and serum samples from CRC patients implicate this lncRNA as a functional regulator of tumor development, acting in the dysregulated migratory and proliferative capacities of tumor cells. The current study also suggests a novel role of circulating NORAD in distinguishing CRC from benign diseases and highlights its potential role in clinical diagnosis and differentiation of CRC. Thus, NORAD holds promise as a biomarker and/or therapeutic target for CRC. Further investigations are required to elucidate the complete underlying mechanisms of NORAD in tumorigenesis.

\section{Acknowledgments}

This work was supported by National Natural Science Foundation of China (No. 81501819 and 81472025),
Shandong Technological Development Project (STDP, 2015GSF118167), the Outstanding Young Scientist Research Award Fund of Shandong Province (BS2014YY002), Taishan Scholar Program of Shandong Province; Natural Science of Basic Scientific Research Foundation of Shandong University, Grant/Award Number: 2017BTS01.

\section{Author contributions}

WL, DL, and WC designed the study and wrote the manuscript; WL conducted the experiments and contributed to the analysis of data. YS and XY contributed to samples collection and RNA extraction; DW assisted inpatient recruitment and contributed to the analysis of data. All authors approved the final version for publication. All authors contributed to data analysis, drafting and revising the article, gave final approval of the version to be published, and agree to be accountable for all aspects of the work.

\section{Disclosure}

The authors report no conflicts of interest in this work.

\section{References}

1. Siegel RL, Miller KD, Jemal A. Cancer statistics, 2016. CA Cancer J Clin. 2016;66(1):7-30.

2. Torre LA, Bray F, Siegel RL, Ferlay J, Lortet-Tieulent J, Jemal A. Global cancer statistics, 2012. CA Cancer J Clin. 2015;65(2):87-108.

3. Brenner H, Kloor M, Pox CP. Toward better control of colorectal cancer. Lancet. 2014;383(9927):1490-1502.

4. Figueredo A, Coombes ME, Mukherjee S. Adjuvant therapy for completely resected stage II colon cancer. Cochrane Database Syst Rev. 2008;3(3):CD005390.

5. Huarte M. The emerging role of lncRNAs in cancer. Nat Med. 2015; 21(11):1253-1261.

6. Rinn JL, Chang HY. Genome regulation by long noncoding RNAs. Annu Rev Biochem. 2012;81:145-166.

7. Han D, Wang M, Ma N, Xu Y, Jiang Y, Gao X. Long noncoding RNAs: novel players in colorectal cancer. Cancer Lett. 2015;361(1):13-21.

8. Schmitt AM, Chang HY. Long Noncoding RNAs in Cancer Pathways. Cancer Cell. 2016;29(4):452-463.

9. Ulitsky I, Bartel DP. lincRNAs: genomics, evolution, and mechanisms. Cell. 2013;154(1):26-46.

10. Smolle M, Uranitsch S, Gerger A, Pichler M, Haybaeck J. Current status of long non-coding RNAs in human cancer with specific focus on colorectal cancer. Int J Mol Sci. 2014;15(8):13993-14013.

11. Hirata H, Hinoda $Y$, Shahryari V, et al. Long noncoding RNA MALAT1 promotes aggressive renal cell carcinoma through Ezh2 and interacts with miR-205. Cancer Res. 2015;75(7):1322-1331.

12. Svoboda M, Slyskova J, Schneiderova M, et al. HOTAIR long noncoding RNA is a negative prognostic factor not only in primary tumors, but also in the blood of colorectal cancer patients. Carcinogenesis. 2014;35(7):1510-1515.

13. Su J, Zhang E, Han L, et al. Long noncoding RNA BLACAT1 indicates a poor prognosis of colorectal cancer and affects cell proliferation by epigenetically silencing of p15. Cell Death Dis. 2017;8(3):e2665.

14. Lee S, Kopp F, Chang TC, et al. Noncoding RNA NORAD regulates genomic stability by sequestering PUMILIO proteins. Cell.2016;164(1-2): 69-80.

15. Tichon A, Gil N, Lubelsky Y, et al. A conserved abundant cytoplasmic long noncoding RNA modulates repression by Pumilio proteins in human cells. Nat Commun. 2016;7:12209. 
16. Tichon A, Perry RB, Stojic L, Ulitsky I. SAM68 is required for regulation of Pumilio by the NORAD long noncoding RNA. Genes Dev. 2018;32(1):70-78.

17. Li H, Wang X, Wen C, et al. Long noncoding RNA NORAD, a novel competing endogenous RNA, enhances the hypoxia-induced epithelialmesenchymal transition to promote metastasis in pancreatic cancer. Mol Cancer. 2017;16(1):169.

18. Wu X, Lim ZF, Li Z, et al. NORAD Expression Is Associated with Adverse Prognosis in Esophageal Squamous Cell Carcinoma. Oncol Res Treat. 2017;40(6):370-374.

19. Liu H, Li J, Koirala P, et al. Long non-coding RNAs as prognostic markers in human breast cancer. Oncotarget. 2016;7(15):20584-20596.

20. Ventura A. NORAD: Defender of the Genome. Trends Genet. 2016; 32(7):390-392.
21. Zhang J, Li XY, Hu P, Ding YS. LncRNA NORAD contributes to colorectal cancer progression by inhibition of miR-202-5p. Oncol Res. 2018. Epub 2018 Feb 22.

22. Guda K, Veigl ML, Varadan V, et al. Novel recurrently mutated genes in African American colon cancers. Proc Natl Acad Sci U S A. 2015; 112(4):1149-1154.

23. Vaiopoulos AG, Athanasoula KCH, Papavassiliou AG. Epigenetic modifications in colorectal cancer: molecular insights and therapeutic challenges. Biochim Biophys Acta. 2014;1842(7):971-980.

24. Bardhan K, Liu K. Epigenetics and colorectal cancer pathogenesis. Cancers (Basel). 2013;5(2):676-713. 


\section{Supplementary material}

Table SI Sequences of qPCR primers and siRNAs

\begin{tabular}{l|l}
\hline qPCR primer name & Sequence, $\mathbf{5}^{\prime} \mathbf{- 3} \mathbf{3}^{\prime}$ \\
\hline NORAD (forward) & AGCGAAGTCCCGAACGACGA \\
NORAD (reverse) & TGGGCATTTCCAACGGGCCAA \\
GAPDH (forward) & ACCCACTCCTCCACCTTTGAC \\
GAPDH (reverse) & TGTTGCTGTAGC CAAATTCGTT \\
siRNA name & Sequence, 5'-3' \\
si-NORAD (No I) & GCGGTTGGTCTTCATTCTA \\
si-NORAD (No 2) & GCTAGTGAATTAGCCTATT \\
si-NORAD (No 3) & GCTAGTGAATTAGCCTATT \\
\hline
\end{tabular}

Note: The si-negative control was obtained from Ribobio (Guangzhou China).

Abbreviations: qPCR, Quantitative real-time PCR; siRNA, Small interfering RNA.

\section{Publish your work in this journal}

OncoTargets and Therapy is an international, peer-reviewed, open access journal focusing on the pathological basis of all cancers, potential targets for therapy and treatment protocols employed to improve the management of cancer patients. The journal also focuses on the impact of management programs and new therapeutic agents and protocols on

patient perspectives such as quality of life, adherence and satisfaction. The manuscript management system is completely online and includes a very quick and fair peer-review system, which is all easy to use. Visit http://www.dovepress.com/testimonials.php to read real quotes from published authors. 